\title{
CARACTERIZAÇÃO DE RESISTÊNCIA À FADIGA DE AÇO SAE 1008 E ALUMÍNIO 1200 H14 UTILIZANDO FLEXÃO ALTERNADA COM CORPOS DE PROVA NORMALIZADOS E PROPOSTOS*
}

Gustavo Henrique Bolognesi Donato ${ }^{1}$ Rodrygo Figueiredo Moço

\section{Resumo}

Frente ao crescente número de falhas por fadiga, o projeto considerando cargas cíclicas passou a ser uma fase natural da concepção e otimização de componentes, em especial de setores de responsabilidade, como aeronáutico, automobilístico e de óleo e gás. Em geral, as avaliações se baseiam em resultados de ensaios uniaxiais que empregam máquinas universais servo-controladas de tração-compressão ou tração-tração. Entretanto, embora precisos, tais equipamentos custam centenas de milhares de dólares, apresentam elevado custo operacional e de manutenção e exigem expressivo tempo para a realização dos ensaios. Como alternativas simples e baratas existem as máquinas do tipo flexão alternada (biela-manivela), as quais podem apresentar curvas de vida à fadiga comparáveis ao caso uniaxial se os campos de deformações e tensões nas amostras forem bem conhecidos. Dessa forma, buscando avançar nesse tópico e fazendo uso de refinados modelos não lineares de elementos finitos, este artigo apresenta uma metodologia de ensaios de flexão alternada controlada por deformação (curvas $\varepsilon-\mathrm{N}$ ), assim como uma análise crítica dos resultados experimentais. Foram ensaiados um aço SAE 1008 e um alumínio 1200 H14 e, além da geometria recomendada pela ASTM B593, uma nova geometria é proposta para menor dispersão dos resultados experimentais. A metodologia desenvolvida obteve sucesso na condução dos ensaios e a geometria proposta reduziu a dispersão dos resultados para o aço SAE 1008 que apresenta elevado encruamento. As curvas $\varepsilon$ - $N$ obtidas, além de diretamente úteis para projetos mecânicos, apoiarão comparações futuras com resultados de fadiga uniaxial dos mesmos materiais.

Palavras-chave: Ensaio de fadiga; Flexão alternada; Aço SAE 1008; Alumínio série 1000.

\section{FATIGUE STRENGTH ASSESSMENT OF A SAE 1008 STEEL AND A 1200 H14 ALUMINUM BY ALTERNATING BENDING FATIGUE TESTS USING STANDARDIZED AND PROPOSED SAMPLES}

\begin{abstract}
As a response to the growing number of fatigue failures, the assessment of cyclic loading has become a natural phase of the design and optimization of high responsibility components, such as those from oil and gas, aeronautical and automotive areas. These assessments are generally based on uniaxial testing, which employ tension-compression or tension-tension servo-controlled universal machines. However, while accurate, such machines are costly and time-consuming for conducting each test. The alternating bending fatigue machine is a simple and less expensive solution, which can lead to fatigue curves comparable to the uniaxial ones, once the stress and strain fields at the sample are well known. Thus, as a step forward to better understand this subject, this paper develop very refined nonlinear finite element models for selected samples' geometries and presents the methodology and respective results for strain controlled alternating bending fatigue tests ( $\varepsilon-\mathrm{N}$ curves). Two materials were tested (SAE 1008 steel and $1200 \mathrm{H} 14$ aluminum), and besides the geometry recommended by the ASTM B593, a new geometry was proposed for reducing scatter of experimental results. The tests could be well conducted using the developed methodology and the proposed geometry presents less scatter for the SAE 1008 steel, which presents high hardening exponent. The obtained $\varepsilon-\mathrm{N}$ curves are not only useful for mechanical design, but will also support future comparisons with uniaxial fatigue results of the same materials.
\end{abstract}

Keywords: Fatigue test; Alternating bending; SAE 1008 steel; 1000 series aluminum.

1 Engenheiro Mecânico, Doutor, Docente do Departamento de Engenharia Mecânica do Centro Universitário da FEl, São Bernardo do Campo, SP, Brasil, gdonato@fei.edu.br..

2 Engenheiro Mecânico, Aluno de Mestrado do Departamento de Engenharia Mecânica do Centro Universitário da FEI, São Bernardo do Campo, SP, Brasil. 


\section{INTRODUÇÃO}

O fenômeno de fadiga é definido pela American Society for Testing and Materials (ASTM) [1] como um "processo de degradação estrutural progressiva, permanente e localizada que ocorre em materiais submetidos a tensões e deformações flutuantes em um ou mais pontos e que podem culminar em trincas ou fratura completa após um número suficiente de ciclos". Portanto, o processo de fadiga é irreversível, cumulativo e está sempre atrelado à existência de flutuações nos carregamentos aplicados. Assim, em estruturas cujos carregamentos incluam flutuações, as verificações da mecânica dos sólidos clássica devem ser complementadas por verificações de vida à fadiga [2], em especial para componentes críticos como das indústrias aeronáutica, automotiva, nuclear e de petróleo e gás. Tais considerações permitem ao engenheiro estimar a vida de uma estrutura para a qual não ocorrerá a falha por formação de microdefeitos (trincas) e seu crescimento até a fratura [3,4].

Dessa forma, as propriedades à fadiga dos materiais obtidas a partir de amostras laboratoriais devem ser precisas, de forma a permitir a transferência de propriedades mecânicas para estruturas reais. Na maioria dos casos, os ensaios são uniaxiais e empregam máquinas universais servo-controladas que submetem corpos de provas a solicitações de tração-compressão ou tração-tração. Entretanto, embora precisos, tais equipamentos custam centenas de milhares de dólares, apresentam elevado custo operacional e de manutenção e exigem expressivo tempo para a realização dos ensaios. Como alternativas mais simples e baratas existem as máquinas do tipo flexão alternada (biela-manivela). As curvas de vida à fadiga obtidas a partir destas (especialmente as curvas $\varepsilon-\mathrm{N}$ ), no entanto, podem apresentar algumas divergências se comparadas ao caso uniaxial, especialmente para regimes de baixo ciclo (com existência de plasticidade). Tais discrepâncias não derivam de menor precisão dos equipamentos, mas sim do campo de tensões não uniforme aplicado ao espécime, o que motiva o presente estudo em busca de um melhor entendimento destes ensaios. Além disso, a geometria sugerida pela ASTM (American Society for Testing and Materials) [6] possui raios de concordância que concentram tensões podendo causar aumento do espalhamento dos resultados experimentais. Dessa forma, esse trabalho objetiva viabilizar a realização e pós-processamento dos resultados de ensaios de fadiga flexional em um aço SAE 1008 e um alumínio 1200 H14 garantindo os campos de deformação impostos, que foram calibrados por meio de modelos de elementos finitos não lineares e refinados, e comparar os resultados obtidos pela geometria normalizada [6] com uma geometria proposta pelos autores ${ }^{1,2}$ com o objetivo de reduzir a dispersão dos resultados experimentais.

\subsection{Fadiga controlada por deformação $(\varepsilon-\mathrm{N})$}

Existem diversos modelos para se descrever o dano causado por fadiga, sendo que para iniciação de trincas se destacam o método $\sigma-\mathrm{N}$ (quando o carregamento é controlado por tensão - $\sigma$ ) e o método $\varepsilon-\mathrm{N}$ (quando o carregamento é controlado por deformação $-\varepsilon-$ caso deste estudo) [7]. Independente da tratativa matemática e do controle dos ensaios, a falha por fadiga, que apresenta natureza intrinsecamente estatística, ocorre após um processo de dano plástico microscópico que culmina na formação de microtrincas, que acabam concentrando tensões e ao mesmo tempo diminuem a seção transversal do material que resiste ao esforço aplicado [3,4].

Um ensaio de fadiga controlado por deslocamento (e consequentemente deformação) significa que uma amplitude de deformação constante é imposta à amostra até a falha 
por fadiga [3]. A Figura 1(a) ilustra um carregamento senoidal típico de ensaios controlados por deformação, sendo nesta definidas as principais grandezas envolvidas, tais como amplitude de deformação $\left(\varepsilon_{a}\right)$, deformação média $\left(\varepsilon_{m}\right)$, ciclo e reversão, além das deformações máxima $\left(\varepsilon_{\text {máx }}\right)$ e mínima $\left(\varepsilon_{\text {mín }}\right)$. Outra grandeza de grande interesse aos ensaios de fadiga é a razão de carregamento, dada neste caso pela equação 1. Um carregamento com tensão média zero apresenta $R=-1$ e é dito completamente reverso [4]. Quanto maior for o $\mathrm{R}$, mais trativo é o carregamento e reduções na vida em fadiga são esperadas [3].

$$
R=\frac{\varepsilon_{\text {min }}}{\varepsilon_{\text {máx }}}
$$

Uma das vantagens do uso de ensaios controlados por deformação é a possibilidade de se caracterizar os materiais sob regimes elasto-plásticos, já que a amplitude de deformação $\varepsilon_{a}$ incorporara parcelas elásticas e plásticas, conforme apresentado na Figura $1(b)$ pela curva tensão $(\sigma)$ vs. deformação $(\varepsilon)$ típica de um material metálico.

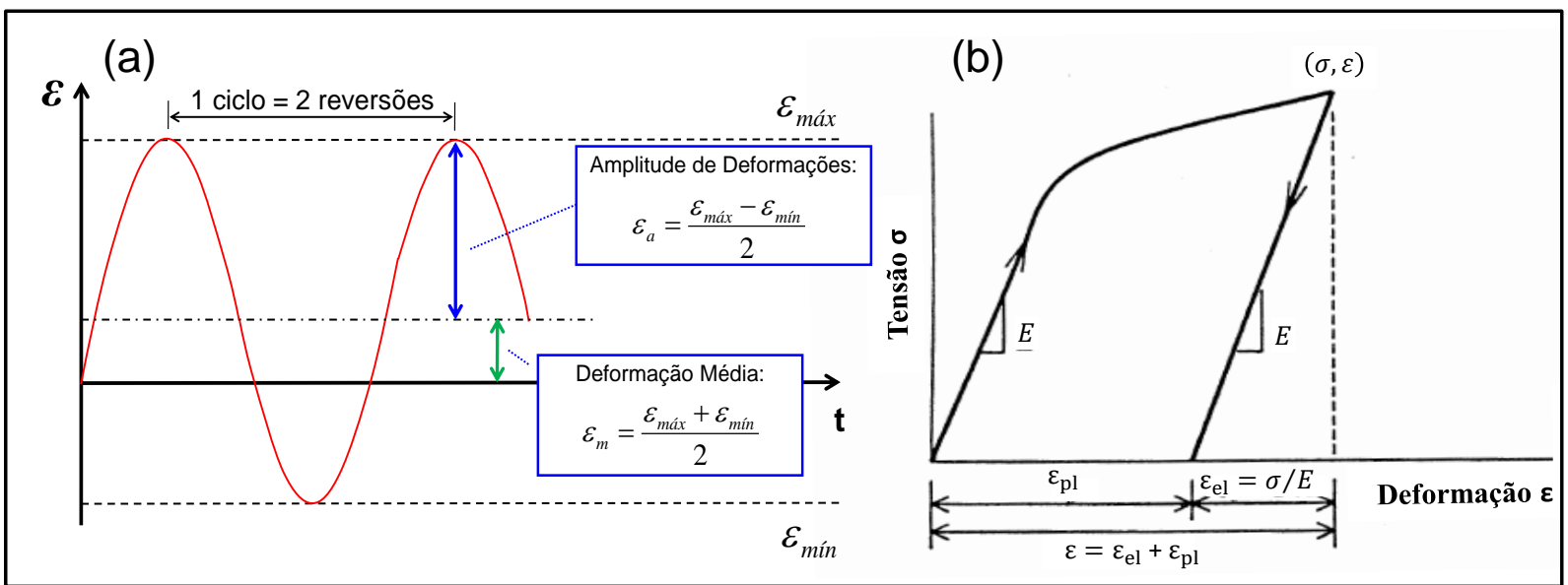

Figura 1. (a) Carregamento senoidal controlado por deformação. (b) Curva tensão $\sigma$ vs. deformação $\varepsilon$ típica de um material metálico.

O resultado de um ensaio controlado sob uma dada amplitude de deformação $\left(\varepsilon_{a}\right)$ é um número respectivo de ciclos à falha $\left(\mathrm{N}_{\mathrm{f}}\right)$. Um grupo de ensaios permite a obtenção da chamada curva $\varepsilon-\mathrm{N}$, a qual é costumeiramente apresentada como na Figura 2 (curva total) em termos de $\log \left(\varepsilon_{a}\right)$ vs. $\log \left(2 \mathrm{~N}_{\mathrm{f}}\right)$. Esta curva é usualmente bem representada para metais pelo modelo de Coffin-Manson (equação 2).

$$
\varepsilon_{a}=\varepsilon_{a-e l}+\varepsilon_{a-p l}=\frac{\sigma_{f}^{\prime}}{E}\left(2 N_{f}\right)^{b}+\varepsilon_{f}^{\prime}\left(2 N_{f}\right)^{c}
$$

Na Equação $2 \varepsilon_{a-e l}$ é a parcela elástica da amplitude de deformação, $\varepsilon_{a-p l}$ é a parcela plástica da amplitude de deformação, $\sigma_{f}^{\prime}$ é o coeficiente de resistência à fadiga, $b$ é o expoente de resistência à fadiga, $\varepsilon_{f}^{\prime}$ é o coeficiente de ductilidade à fadiga e $c$ é 0 expoente de ductilidade à fadiga. A Figura 2 ilustra também as curvas das parcelas elástica e plástica apresentadas separadamente. É possível observar que para grandes deformações e baixo número de ciclos a deformação plástica é predominante e que em pequenas deformações e alto número de ciclos a deformação elástica predomina. O ponto de intersecção entre as retas das duas parcelas é conhecido como "vida de transição - $2 \mathrm{~N}_{\mathrm{t}}$ " e delimita, para o material em ensaio, o regime de baixo 
ciclo (predomínio de plasticidade) e o regime de alto ciclo (predomínio de elasticidade) [4].

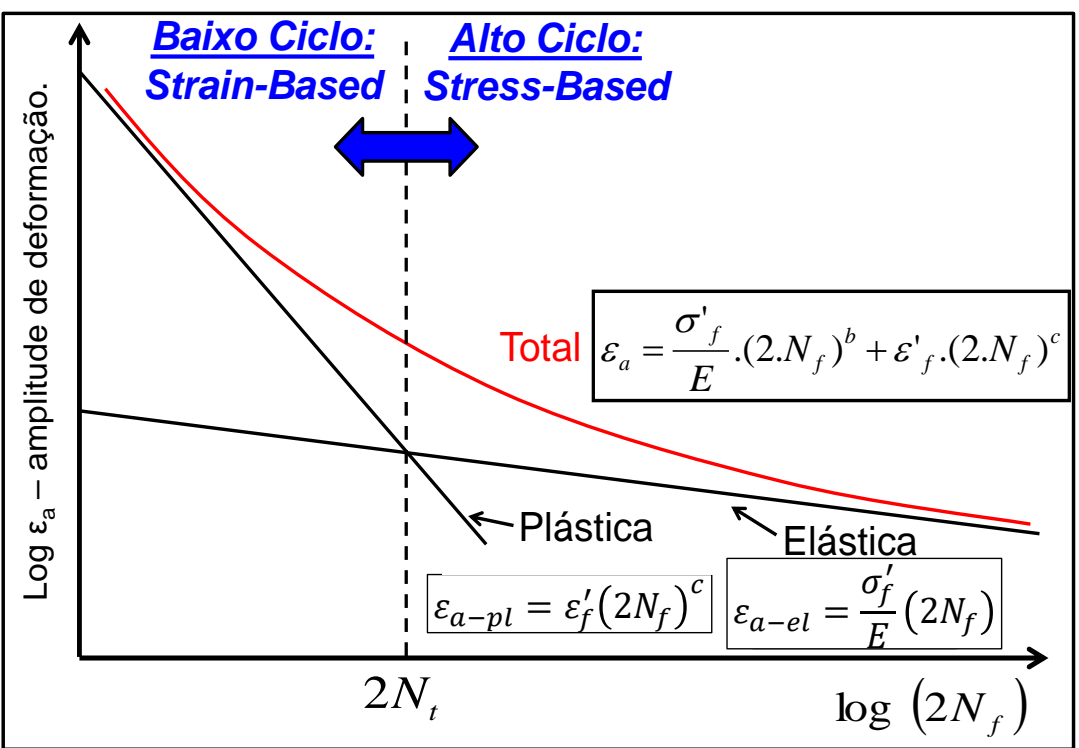

Figura 2. Modelo de Coffin-Manson para uma curva típica obtida em ensaio de fadiga controlado por deformação.

\subsection{Geometria padronizada pela ASTM para fadiga flexional alternada}

A geometria normalizada pela ASTM B593 [6] é apresentada pela Figura 3(a) e apresenta diversas limitações, a saber: i) a referida norma somente se aplica a ligas de cobre, embora aplicada por alguns pesquisadores a outros materiais [9]; ii) 0 desenvolvimento de tal geometria é baseado na teoria de isoflexão da mecânica dos sólidos, não incorporando os efeitos de concentração de tensões dos raios de concordância das extremidades da área útil; iii) consequentemente, a falha pode ocorrer na área central (útil) ou nos concentradores de tensões, podendo caracterizar indesejável espalhamento experimental dos dados de laboratório. Portanto, em paralelo ao desenvolvimento de uma metodologia de ensaio $\varepsilon-\mathrm{N}$ robusta com base em modelos de elementos finitos refinados e que incorporem plasticidade, a proposição de uma geometria que busque minimizar tais limitações emerge como interesse do trabalho.

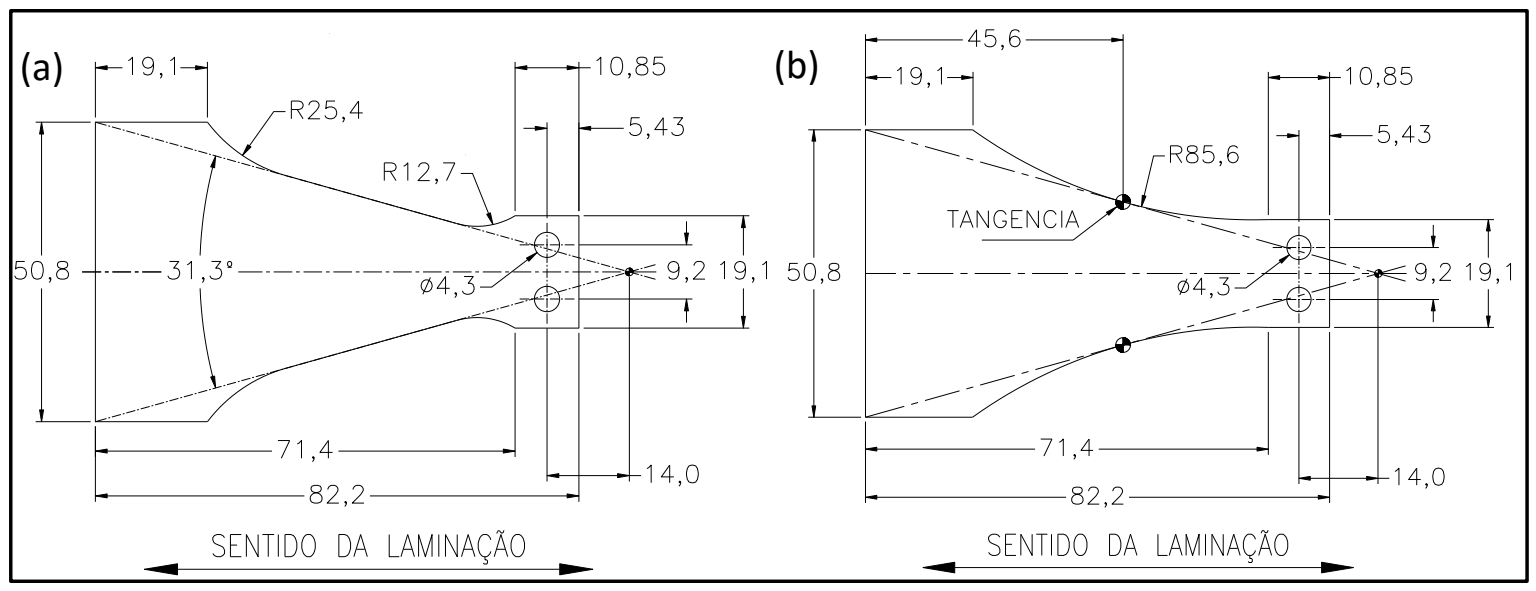

Figura 3. Desenho esquemático das geometrias (a) normalizada e (b) proposta. 


\section{MATERIAIS E MÉTODOS}

\subsection{Materiais em análise}

Os corpos de prova foram usinados a partir de chapas de $1,5 \mathrm{~mm}$ de espessura de aço SAE 1008 e alumínio $1200 \mathrm{H14}$, com as composições químicas das Tabelas 1 e 2 respectivamente. Para a obtenção das propriedades mecânicas foram realizados ensaios de tração de acordo com a norma ASTM E8/E8M [5]. O aço apresentou $E=$ $187 \mathrm{GPa}$, tensão limite de escoamento $\mathrm{S}_{\mathrm{LE}}=207 \mathrm{MPa}$ e tensão limite de resistência $S_{L R}=331 \mathrm{MPa}$, já para o alumínio $\mathrm{E}=71 \mathrm{GPa}, \mathrm{S}_{\mathrm{LE}}=114 \mathrm{MPa}$ e $\mathrm{S}_{\mathrm{LR}}=121 \mathrm{MPa}$.

Tabela 1. Composição química do aço SAE 1008

\begin{tabular}{c|c|c|c|c|c|c|c}
\hline $\begin{array}{c}\text { Materiais } \\
\text { presentes }\end{array}$ & $\begin{array}{c}\text { Carbono } \\
(\mathrm{C})\end{array}$ & $\begin{array}{c}\text { Silício } \\
(\mathrm{Si})\end{array}$ & $\begin{array}{c}\text { Manganês } \\
(\mathrm{Mn})\end{array}$ & $\begin{array}{c}\text { Fosforo } \\
(\mathrm{P})\end{array}$ & $\begin{array}{c}\text { Enxofre } \\
(\mathrm{S})\end{array}$ & $\begin{array}{c}\text { Cromo } \\
(\mathrm{Cr})\end{array}$ & $\begin{array}{c}\text { Titânio } \\
(\mathrm{Ti})\end{array}$ \\
\hline (\% peso) & 0,0717 & 0,0100 & 0,3500 & 0,0160 & 0,0060 & 0,0090 & 0,5300 \\
\hline
\end{tabular}

Tabela 2. Composição química do alumínio $1200 \mathrm{H} 14$.

\begin{tabular}{c|c|c|c|c|c|c|c|c}
\hline $\begin{array}{c}\text { Materiais } \\
\text { presentes }\end{array}$ & $\begin{array}{c}\text { Alumínio } \\
(\mathrm{Al})\end{array}$ & $\begin{array}{c}\text { Cobre } \\
(\mathrm{Cu})\end{array}$ & $\begin{array}{c}\text { Ferro } \\
(\mathrm{Fe})\end{array}$ & $\begin{array}{c}\text { Zinco } \\
(\mathrm{Zn})\end{array}$ & $\begin{array}{c}\text { Manganês } \\
(\mathrm{Mn})\end{array}$ & $\begin{array}{c}\text { Gálio } \\
(\mathrm{Ga})\end{array}$ & $\begin{array}{c}\text { Silício } \\
(\mathrm{Si})\end{array}$ & $\begin{array}{c}\text { Titânio } \\
(\mathrm{Ti})\end{array}$ \\
\hline (\% peso) & 99,37 & 0,01 & 0,30 & 0,01 & 0,01 & 0,01 & 0,32 & 0,01 \\
\hline
\end{tabular}

\subsection{Geometrias propostas}

À luz dos argumentos apresentados na seção 1.2, foi realizado um estudo inicial baseado na mecânica dos sólidos a respeito da área útil em isoflexão da amostra normalizada da figura 3(a). O objetivo era substituir a geometria trapezoidal por uma geometria de raio contínuo que pudesse minimizar as tensões nas extremidades, garantindo leve concentração de tensões no centro da amostra. Esta concentração deveria existir para induzir a falha na região desejada, mas ser o mais reduzida possível (com fator de concentração $K_{t}$ tendendo a 1). Rotinas de otimização e modelos iterativos não-lineares de elementos finitos conduziram à geometria da Figura 3(b), cujos resultados de campos de tensões são apresentados adiante.

\subsection{Modelos numéricos para a deformada do ensaio de fadiga}

Modelos de elementos finitos refinados e não lineares foram elaborados e processados respectivamente em programas MSC Patran 2013 e Marc 2010 para obtenção da distribuição das tensões e deformações de von Mises nas geometrias normalizada (Figura 3(a)) e proposta (Figura 3(b)) para ambos os materiais para as flechas do ensaio de fadiga. Foram usadas malhas com 35000 elementos hexaédricos de oito nós por modelo e o carregamento empregou deslocamentos incrementais de $0,127 \mathrm{~mm}$ até totalizar $12,7 \mathrm{~mm}$, que é a máxima flecha aplicável na máquina utilizada nos ensaios experimentais. A curva tensão-deformação verdadeira experimental foi fornecida como propriedade de material e a teoria de plasticidade incremental $J_{2}$ foi utilizada. A Figura 4 ilustra a malha, vinculação e carregamento. 

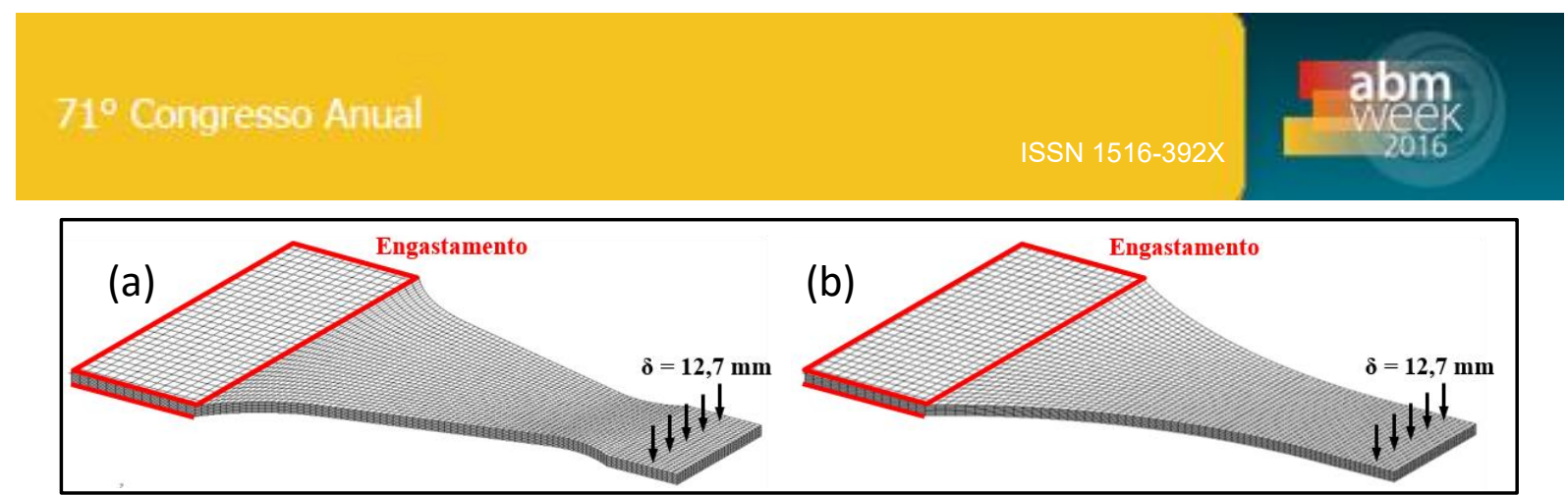

Figura 4. Modelos de elementos finitos das geometrias (a) normalizada e (b) proposta.

\subsection{Usinagem dos corpos de prova}

Inicialmente foram cortados 100 "blanks" retangulares a partir de chapas de 1,5 mm de espessura. Na sequência, 4 grupos de 25 "blanks" foram empilhados e montados em dispositivos especialmente desenvolvidos (Figura 5(a)) com chapas de aço laminadas a quente de $3 / 4$ " de espessura para que a usinagem pudesse ser feita de uma só vez em centro de usinagem com mesmo dimensional para todas as amostras. Uma vez usinados os corpos de provas foram lixados nas faces e laterais com lixas de granulometria 200 até 600 , e então polidos com pastas de diamante de $6 \mu \mathrm{m}$ até $1 \mu \mathrm{m}$ (Figura 5(b)). Foi atingida a rugosidade $\mathrm{Ra}=0,30 \pm 0,10 \mu \mathrm{m}$.

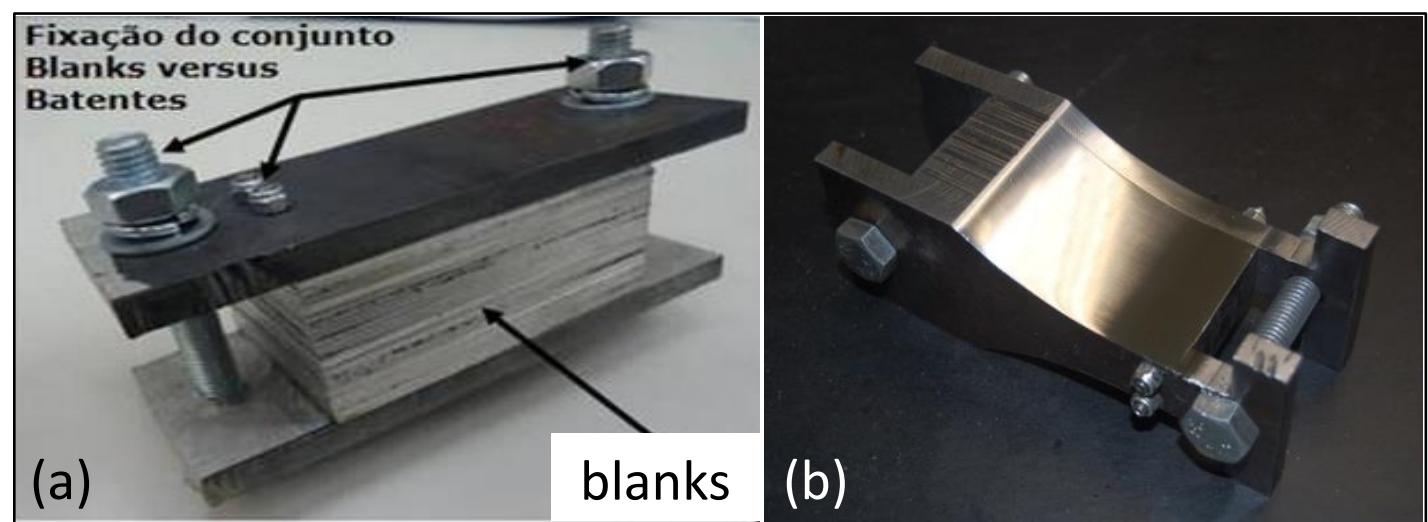

Figura 5. (a) Dispositivo de fixação das chapas para usinagem. (b) Corpos de prova de geometria proposta após o polimento.

\subsection{Procedimento experimental}

Os ensaios em controle de deformação foram realizados em uma máquina de fadiga flexional disponível no CDMatM-FEI, a qual foi potencializada com instalação de célula de carga e variador de frequência para controle da frequência de ensaio (Figura 6(a)). A maior dificuldade é na preparação do ensaio, para que a flecha desejada na amostra seja devidamente ajustada na máquina. Para auxiliar nisto, o equipamento foi modelado computacionalmente (Figura 6(b)) e foi estudado em detalhes o seu cinematismo, incluindo os deslocamentos transversais impostos na amostra e longitudinais da biela, considerando os efeitos de grandes deslocamentos.

Usando os resultados de modelos de elementos finitos (apresentados adiante) eram conhecidos os valores de flecha $\delta$ a impor buscando as amplitudes de deformação desejadas. Este procedimento foi calibrado para cada material ensaiado usando célula de carga e amostra instrumentada com strain-gages, como ilustra a Figura 6(c). Os erros ficaram abaixo de $0,5 \%$. Todos os ensaios foram realizados com razão de carga $\mathrm{R}=-1$ (carga completamente reversa) e o número de ciclos à falha foi obtido por contador digital de rotações da marca Novus que foi adquirido e instalado em definitivo no equipamento. 

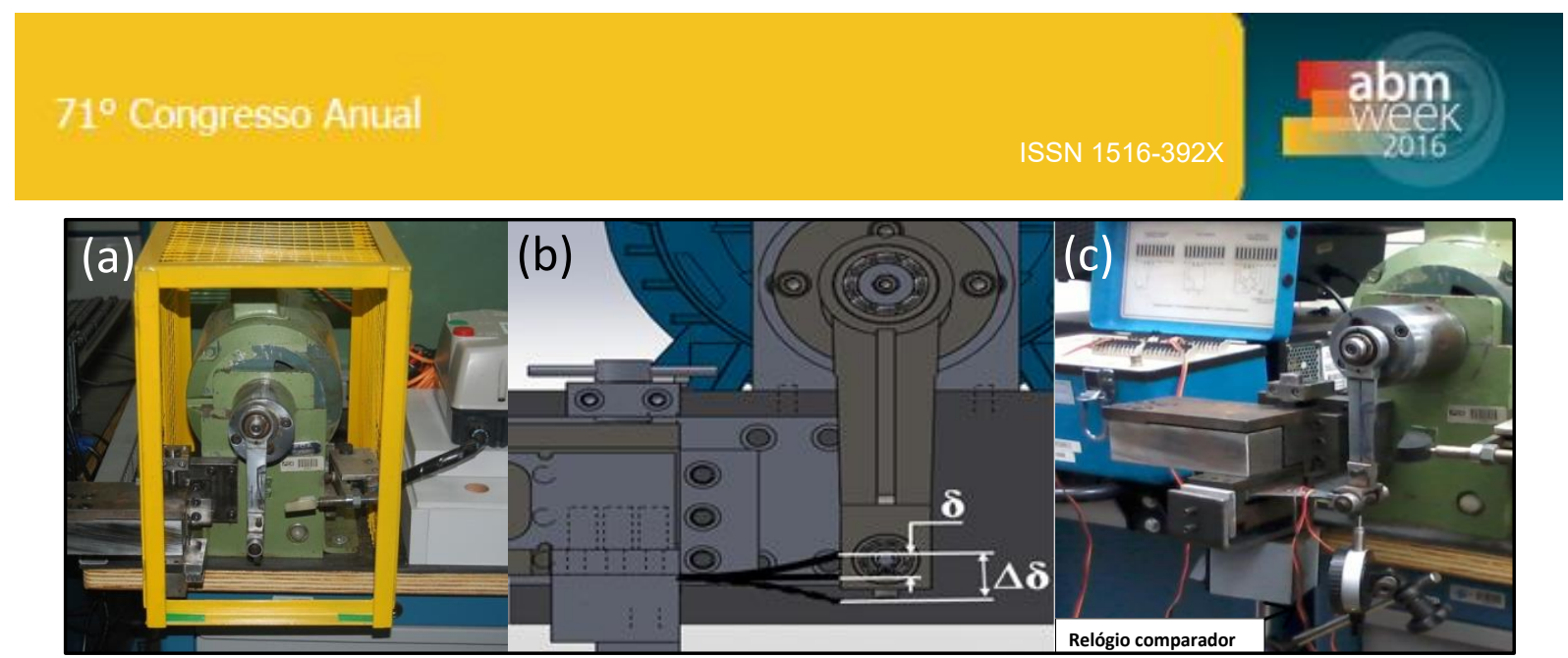

Figura 6. (a) Máquina de fadiga flexional. (b) Modelagem do equipamento. (c) Ilustração do procedimento para calibração da amplitude de deformação aplicada.

\section{RESULTADOS E DISCUSSÃO}

\subsection{Resultados numéricos}

As Figuras 7(a) e 7(c) apresentam as deformações de von Mises para a geometria da ASTM B593 [6] sob $\delta=5 \mathrm{~mm}$ respectivamente considerando aço e alumínio. As Figuras 7 (b) e 7(d) apresentam as deformações respectivas para a geometria proposta. Fica evidente que a concentração de deformações na geometria proposta ocorre na região central da amostra e de maneira mais progressivamente distribuída como originalmente objetivado. A concentração de deformações no engastamento das duas geometrias é fruto da estratégia de vinculação, mas deve ser notada, pois gerou alguns problemas no alumínio.

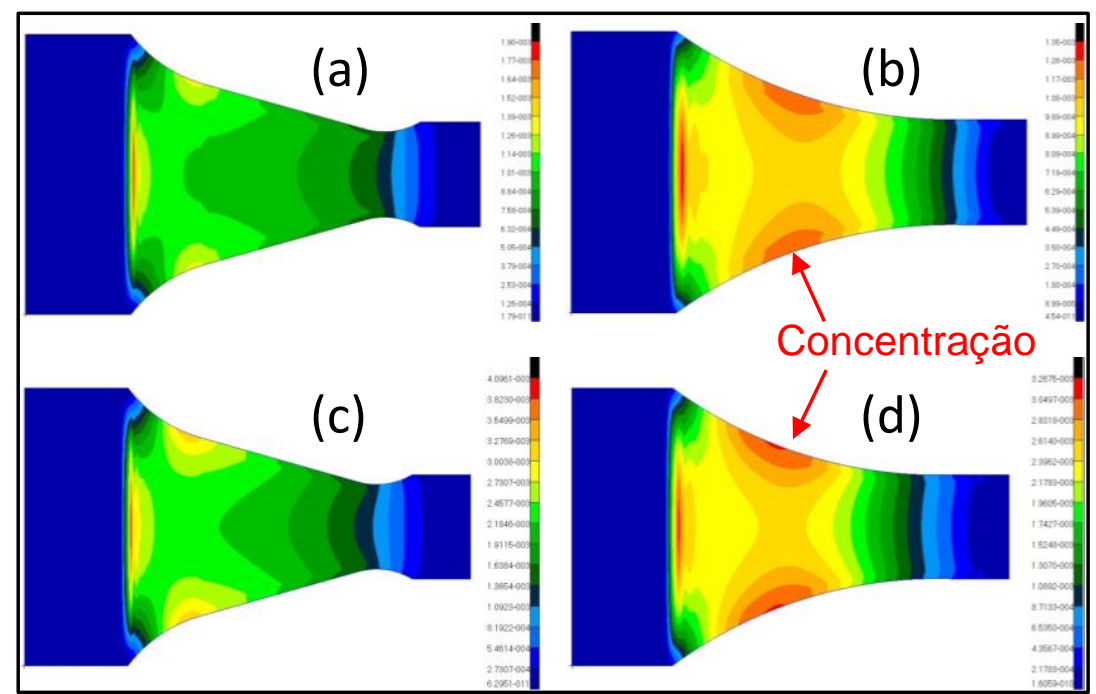

Figura 7. Deformações de von Mises nas geometrias da ASTM para (a) aço e (c) alumínio e proposta para (b) aço e (d) alumínio.

Com base no tratamento dos dados obtidos pelas simulações numéricas foi possível obter a evolução das tensões e deformações máximas de von Mises em função da flecha imposta $\delta$ para cada material e geometria. A Figura 8 possui um exemplo de polinômio de ajuste (para amostra proposta feita em aço) que facilita a preparação da máquina e garante a imposição das amplitudes de deformação desejadas com erros inferiores a $0,5 \%$ quando verificadas com extensômetros elétricos de resistência (strain-gages). Todo o trabalho experimental realizado está baseado em evoluções como a apresentada. 


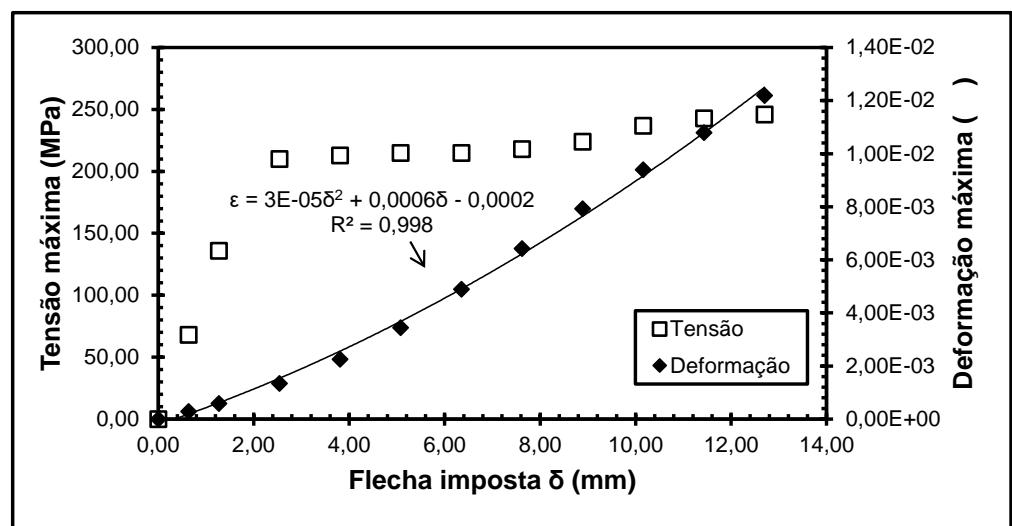

Figura 8. Relação entre tensão, deformação e flecha para a geometria proposta de aço.

\subsection{Resultados experimentais}

A Figura 9 apresenta todos os resultados de fadiga para o aço SAE 1008 utilizando a geometria normalizada $(\mathrm{N})$ e proposta $(\mathrm{P})$. Os marcadores losangulares representam a amplitude de deformação total imposta $v s$. o número de ciclos à falha, enquanto os marcadores triangulares e quadrados representam respectivamente as parcelas plástica e elástica. Fica evidente que os resultados advindos de ambas as geometrias se ajustaram bem às formulações das parcelas elástica e plástica do modelo de CoffinManson. A geometria proposta, em particular, mostrou uma ligeira redução no espalhamento experimental (o que foi reforçado pela análise estatística apresentada a seguir). Além disso, é possível perceber grande proximidade do comportamento elástico e ligeira subestimativa de vida no regime plástico quando empregada a amostra com geometria proposta.

É importante ressaltar que em ambas as geometrias os pontos correspondentes a "vida infinita" para os quais os ensaios foram interrompidos em 11 milhões de ciclos não foram utilizados nem para a adequação da linha de tendência e nem para a análise estatística. $O$ tratamento estatístico dos dados experimentais da Figura 9 foi feito de acordo com a norma ASTM E739 [8] proporcionando as propriedades médias, e também as propriedades relacionadas aos limites inferiores e superiores que abrangem um intervalo de confiança de $95 \%$. Todos os resultados aparecem na Tabela 3 para ambas as geometrias. Fica evidente que para o aço SAE 1008 os desvios percentuais em relação à média são menores para a geometria proposta. 


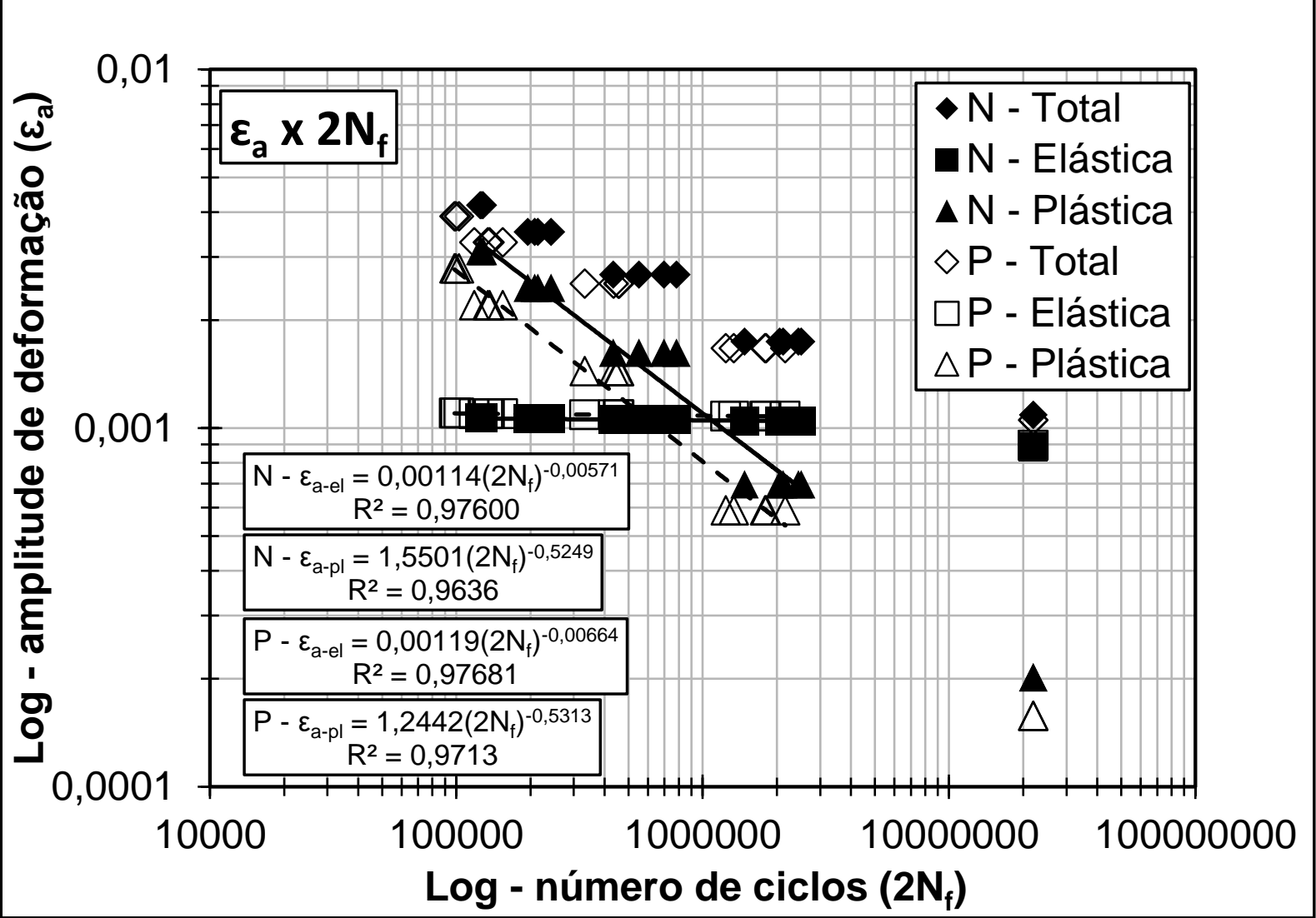

Figura 9. Curvas $\varepsilon-N$ para aço SAE 1008 de ambas as geometrias, assim como representação dos modelamentos das parcelas elástica e plástica da curva total.

Tabela 3. Propriedades de fadiga, com confiança de 95\%, do aço SAE 1008.

\begin{tabular}{c|c|c|c}
\hline \multicolumn{4}{c}{ Aço SAE 1008 (geometria ASTM) } \\
\hline Propriedade & $\begin{array}{c}\text { Limite inferior } \\
\text { (erro \% da média) }\end{array}$ & Média & $\begin{array}{c}\text { Limite superior } \\
\text { erro \% da média) }\end{array}$ \\
\hline b & $0,00597(+4,6 \%)$ & 0,00571 & $0,00561(-1,8 \%)$ \\
\hline$\sigma_{f}^{\prime}$ & $222,66(-0,1 \%)$ & 222,85 & $223,24(+0,2 \%)$ \\
\hline c & $-0,571(+8,8 \%)$ & $-0,525$ & $-0,509(-3,0 \%)$ \\
\hline$\varepsilon_{f}^{\prime}$ & $1,925(+24,2 \%)$ & 1,550 & $1,774(+14,5 \%)$ \\
\hline \multicolumn{4}{c}{ Aço SAE 1008 (geometria proposta) } \\
\hline Propriedade & $\begin{array}{c}\text { Limite inferior } \\
(\text { erro \% da média) }\end{array}$ & Média & $\begin{array}{l}\text { Limite superior } \\
\text { (erro \% da média) }\end{array}$ \\
\hline b & $0,00695(+4,7 \%)$ & 0,00664 & $0,00652(+1,8 \%)$ \\
\hline$\sigma f$ & $232,46(+0,0 \%)$ & 232,46 & $233,04(+0,2 \%)$ \\
\hline c & $-0,574(+8,1 \%)$ & $-0,531$ & $-0,513(-3,4 \%)$ \\
\hline$\varepsilon f$ & $1,478(+18,6 \%)$ & 1,246 & $1,370(+10,0 \%)$ \\
\hline
\end{tabular}

A Figura 10 apresenta todos os resultados de fadiga para o alumínio $1200 \mathrm{H} 14$ utilizando a mesma organização anterior. Fica evidenciado que a aderência das parcelas elástica e plástica do modelo de Coffin-Manson foi muito boa para ambas as geometrias, mas ligeiramente superior para o corpo de prova normalizado pela ASTM. 


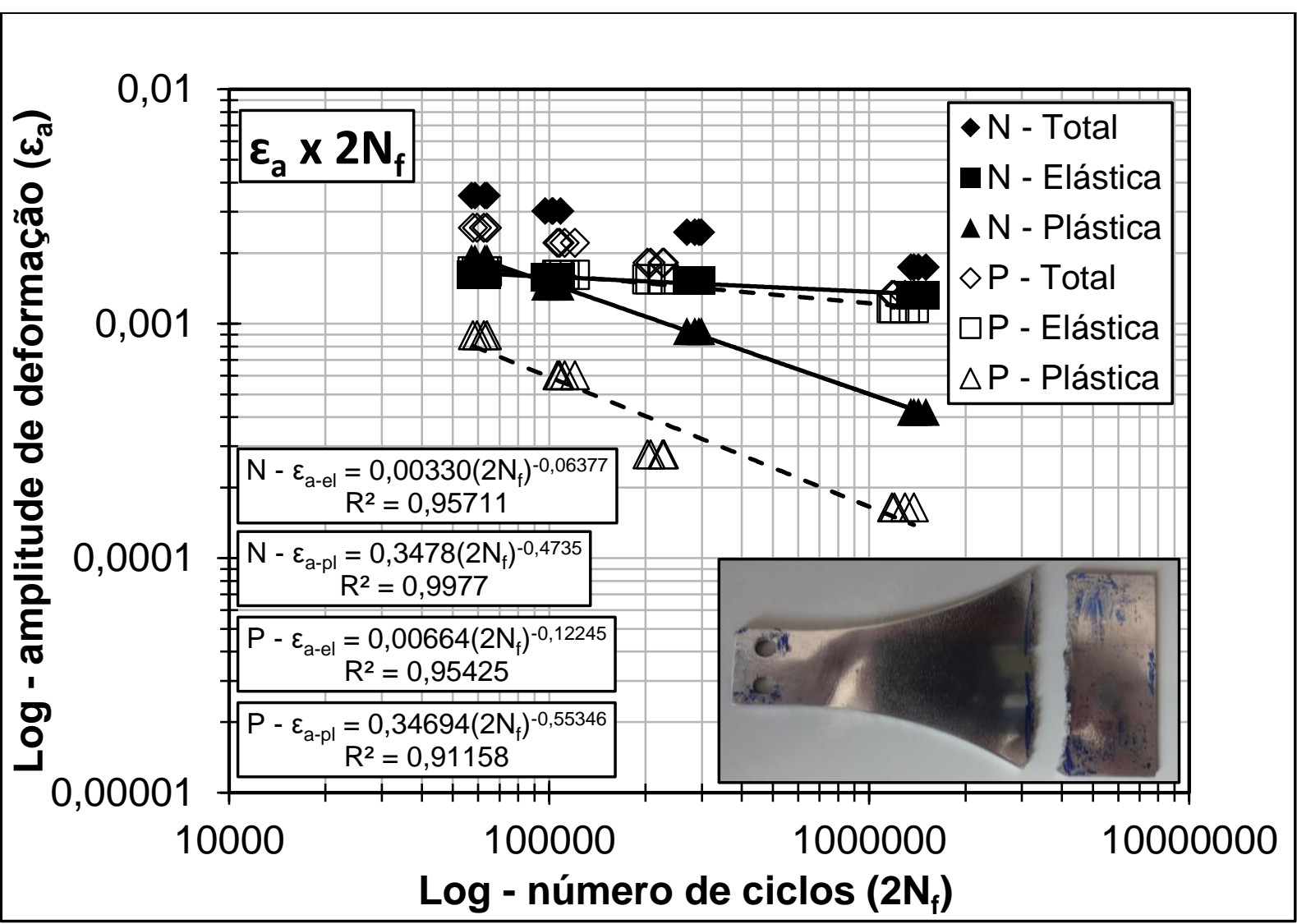

Figura 10. Curvas $\varepsilon-\mathrm{N}$ para o alumínio $1200 \mathrm{H} 14$ de ambas as geometrias, assim como representação dos modelamentos das parcelas elástica e plástica da curva total. $\mathrm{O}$ detalhe presente na figura é a ilustração de um corpo de prova de alumínio que sofreu fratura no engaste.

Tabela 4. Propriedades de fadiga, com confiança de 95\%, do alumínio $1200 \mathrm{H} 14$.

\begin{tabular}{|c|c|c|c|}
\hline \multicolumn{4}{|c|}{ Alumínio 1200 H14 (geometria ASTM) } \\
\hline Propriedade & $\begin{array}{l}\text { Limite inferior } \\
\text { (erro \% da média) }\end{array}$ & Média & $\begin{array}{l}\text { Limite superior } \\
\text { (erro \% da média) }\end{array}$ \\
\hline $\mathrm{b}$ & $0,0757(+18,7 \%)$ & 0,0638 & $0,0586(-8,2 \%)$ \\
\hline$\sigma_{f}^{\prime}$ & $264,26(+9,7 \%)$ & 240,9 & $236,52(-1,8 \%)$ \\
\hline C & $-0,491(+3,6 \%)$ & $-0,474$ & $-0,457(-3,6 \%)$ \\
\hline$\varepsilon_{f}^{\prime}$ & $0,3609(+3,8 \%)$ & 0,3478 & $0,3320(-4,5 \%)$ \\
\hline \multicolumn{4}{|c|}{ Alumínio 1200 H14 (geometria proposta) } \\
\hline Propriedade & $\begin{array}{c}\text { Limite inferior } \\
\text { (erro \% da média) }\end{array}$ & Média & $\begin{array}{l}\text { Limite superior } \\
\text { (erro \% da média) }\end{array}$ \\
\hline$b$ & $\begin{array}{l}-0,1506 \\
(+23,0 \%)\end{array}$ & $-0,1224$ & $-0,1106(-9,6 \%)$ \\
\hline$\sigma_{f}^{\prime}$ & $613,93(+26,7 \%)$ & 484,72 & $454,79(-6,2 \%)$ \\
\hline C & $-0,606(+9,4 \%)$ & $-0,554$ & $-0,587(+6,0 \%)$ \\
\hline$\varepsilon_{f}^{\prime}$ & $0,3892(+12,2 \%)$ & 0,3469 & $0,8730(+151,7 \%)$ \\
\hline
\end{tabular}

Observando a análise estatística na Tabela 4 fica evidente o melhor desempenho da geometria da ASTM [6]. Isto ocorreu pois alguns corpos de prova (em especial para níveis intermediários de plasticidade) apresentaram a falha no engastamento e não na região útil da amostra (detalhe da Figura 10). A explicação para isto deve vir do baixo encruamento apresentado pelo alumínio em estudo (expoente de encruamento 
$\mathrm{n}=0,036 \pm 0,004$ contra $\mathrm{n}=0,206 \pm 0,004$ para o aço SAE 1008) combinado com a tendência de concentração de tensões no engastamento como apresentado pela Figura 7 mesmo para a geometria proposta. Isto, por outro lado, pode ser sanado pelo uso de um raio de concordância ainda único, mas ligeiramente menor, o qual deve concentrar um pouco mais as deformações na região útil da amostra. A geometria da norma ASTM não apresentou esta anomalia pois é reforçada no engaste, embora tenha concentrações de tensão que o trabalho objetivou reduzir. Uma solução a ser tentada no futuro é a combinação das duas abordagens.

Com base nestes resultados, a metodologia e geometria desenvolvidas são recomendadas para materiais de moderado/elevado encruamento e a geometria normalizada é a recomendação atual para os demais materiais.

\section{CONCLUSÕES}

De posse dos resultados obtidos, algumas conclusões centrais emergem:

- As simulações de elementos finitos demonstraram que as distribuições das deformações na geometria proposta localizam o ponto crítico na região desejada e são mais progressivas em relação à geometria da ASTM. Ainda, seus resultados apoiaram diretamente a determinação dos níveis de deslocamentos a usar nos ensaios, favorecendo a precisão dos testes.

- Os resultados obtidos para o aço SAE 1008 são motivadores, na medida em que foi constatado que a dispersão experimental de resultados utilizando a geometria proposta é menor. Ainda, a geometria proposta apresentou boa aderência de resultados ao modelo de Coffin-Manson.

- Para o alumínio 1200 H14 os resultados da geometria proposta foram menos promissores, devido a fraturas no engaste fruto do baixo encruamento. Um novo raio (ou um raio reduzido próximo do engaste) deve ser testado.

- Para ambos os materiais, a geometria proposta gerou ligeira redução da vida em fadiga para uma mesma solicitação aplicada, o que representa uma abordagem a favor da segurança em relação à amostra normalizada e pode ser considerada interessante na prática de avaliação de integridade estrutural.

\section{Agradecimentos}

Este trabalho é suportado pelo Centro Universitário da FEI por meio de recursos materiais, humanos e infraestrutura laboratorial. Adicionalmente, os autores gostariam de agradecer ao CNPQ pela bolsa PIBIC concedida.

\section{REFERÊNCIAS}

[1] American Society for Testing and Materials (ASTM), 2013, "ASTM E1823, Standard Terminology Related to Fatigue and Fracture", Pennsylvania.

[2] Ragab, A., Bayoumi, S. E., 1998. Engineering Solid Mechanics - Fundamentals and Applications. CRC Press: NY

[3] Stephens, R. I., Fatemi, A., Stephens, R. R., Fuchs, H. O., "Metal Fatigue in Engineering". 2nd edition, John Wiley \& Sons, Canada, 2001.

[4] Suresh, S., "Fatigue of Materials". 2nd edition, Cambridge University Press, United Kingdom, 1998.

[5] American Society for Testing and Materials (ASTM), "Standard Test Methods for Tension Testing of Metallic Materials”, ASTM E8/E8M, Philadelphia, 2013. 
[6] American Society for Testing and Materials (ASTM), 2009, "ASTM B593, Standard Test Method for Bending Fatigue Testing for Copper-Alloy Spring Materials", Pennsylvania.

[7] Paul, S. K., Sivaprasat, S., Dhar, S., Tarafder, 2010, "Cyclic plastic deformation and cyclic hardening/softening behavior in 304LN stainless steel", Theoretical and Applied Fracture Mechanics, V. 54.

[8] American Society for Testing and Materials (ASTM), 2010, "ASTM E739, Standard Practice for Statistical Analysis of Linear or Linearized Stress-Life (S-N) and Strain-Life $(\varepsilon-N)$ Fatigue Data", Pennsylvania.

[9] Benedetti, M., Fontanari, V., Scardi, P., Ricardo, C.L.A., Bandini, M., 2009, "Reverse bending fatigue of shot peened 7075-T651 aluminium alloy: The role of residual stress relaxation", International Journal of Fatigue, V. 31, pp. 1225-1236. 